\title{
Ocular Myasthenia Gravis- A rapid review of basics
}

Keywords: Asymmetrical ptosis, ocular myasthenia, anticholinesterase antibodies, generalized disease, thymectomy, corticosteroids

\section{Highlights}

Ocular myasthenia gravis as a unique subset of generalized myasthenia gravis.

Treatment must aim at improvement of ocular symptoms and monitoring for progression to generalized disease.

Individual cases have to be tailored for optimum treatment purpose

\section{Introduction}

Ocular presentation of the postsynaptic defect in neuromuscular transmission occurs almost invariably in every case of myasthenia. Ocular myasthenia gravis is a distinct entity where the weakness is limited to the levator palpebrae superioris, extraocular muscles, and the orbicularis oculi complex. Since the extraocular muscles are not able to adapt to this variable involvement of extraocular muscles, diplopia is a frequent accompaniment to the more common variable ptosis in patients. This presentation can occur in up to $50 \%$ of the patients out of which $50-80 \%$ go on to develop generalized disease. ${ }^{1}$ Usually, about $90 \%$ of patients will go on to develop generalized myasthenia gravis in about 2 years time.,

\section{Pathophysiology}

The tendency of extraocular muscles to be involved earlier than most other muscle groups stems from the higher frequency of synaptic firing in these group of muscles owing to faster development in tension in their twitch fibers leading to earlier fatigue. ${ }^{4}$ Extraocular muscles function as tonic muscles used to sustain gaze in any direction have fewer number of Ach receptors making them more susceptible to loss of receptors. In addition the extraocular muscles represent a clinically distinct form of muscle allotype with several expressing genes being responsible for immune response in an individual.

\section{Ocular manifestations}

Ocular myasthenia can present with any form of pupil sparing motility disorder. ${ }^{5}$ Fluctuating ptosis involving the Levator palpebrae superioris either unilaterally or bilaterally is one of the most common manifestations of myasthenia gravis. Lid fatigue sign, manifested by increased ptosis after prolonged upgaze, and Cogan's lid twitch sign, described as a quick overshooting upward movement followed by a down-drift of the upper lid on returning saccade to primary position from sustained downgaze of about 15 seconds, are commonly seen. Enhanced ptosis of unaffected eye on manual elevation of the ptotic eyelid is another important suggestive feature. Eyelid retraction and "peek sign" on forced eye closure are some of the other useful signs seen. Diplopia due to variable involvement of the extraocular muscles can be seen in any combination although medial rectus and superior rectus muscles are usually the first muscles to be involved. ${ }^{6}$

\section{Diagnosis of ocular myasthenia}

Clinical tests like the improvement of ptosis following ptosis and
Volume 8 Issue 6 - 2018

\author{
Sucharita Ray,' Kamalesh Chakravarty² \\ 'Department of Neurology,All India Institute of Medical \\ Sciences, India \\ ${ }^{2}$ Department of Neurology, Rockland Hospitals, India
}

Correspondence: Sucharita Ray, Senior Resident, Department of Neurology, All India Institute of Medical Sciences, India, Email dr.sucharataray@gmail.com

Received: May 29, 2018 | Published: November 26, 2018

Ice pack test for improvement of ptosis (pack applied for 2 minutes) and ophthalmoplegia (pack held for 5 minutes) can be used routinely. Pharmacologic application of Edrophonium or Neostigmine are sensitive tests that increase the availability of Ach at the synapse, thereby facilitating neuromuscular transmission. Electrophysiological tests include the Repetitive nerve stimulation test (RNST) showing a decremental response in the tested muscle and the Single-fiber electromyography (SFEMG) are used for the detection of postsynaptic neuromuscular junction defect. Of these, SFEMG is the most sensitive diagnostic test for detection of Myasthenia gravis. It has a sensitivity of $85-100 \%$ for ocular myasthenia gravis and a sensitivity $91-100 \%$ in generalized $\mathrm{MG}^{7}{ }^{7}$ Anticholinesterase antibody titers are elevated in up to $80-99 \%$ patients in generalized myasthenia and $30-77 \%$ patients with ocular myasthenia. ${ }^{8}$ About $20 \%$ of generalized myasthenia patients are seronegative for Ach receptor antibodies of which $30 \%$ have antibodies against muscle-specific kinase (anti MuSKAb) expressed on skeletal muscle. ${ }^{4}$ Anti LRP4 antibodies are also found in patients seronegative for acetylcholinesterase and anti-MuSK antibodies and have a milder course of disease with pure ocular forms of presentation.

\section{Treatment of ocular myasthenia gravis}

The aim of treatment is directed at symptomatic improvement of diplopia and ptosis and to monitor for slowing or prevention of progression to generalized myasthenia gravis. Acetylcholinesterase inhibitors used for symptomatic improvement in ocular myasthenia act by increasing the concentration of acetylcholine at the neuromuscular junction. Long-term immunosuppression has been found to be beneficial in preventing generalization of symptoms in ocular myasthenia in up to $75 \% .{ }^{5}$ Steroids are tried in alternate day regimen in mild ocular symptoms not responding to pyridostigmine. Used by itself, prednisone treatment appears to reduce the incidence of generalized myasthenia gravis to $7 \%$ in treated versus $36 \%$ in untreated patients. ${ }^{3}$ Prolonged daily use of steroids mandates the use of a steroid-sparing agent like azathioprine, mycophenolate mofetil, cyclosporine, tacrolimus, cyclophosphamide and the humanized monoclonal antibodies Eculizumab and Belimumab. Thymectomy is advised in patients who present with thymoma as it is believed to delay the onset of generalized symptoms. However in patients without any evidence of thymoma, thymectomy cannot be advised and hence, there are no guidelines for the same. ${ }^{9}$ The task force set by the EFNS for treatment of ocular myasthenia recommended the use of thymectomy only in cases where drug treatment fails and not as first-line treatment. ${ }^{9}$ Class IV recommendations advocate the use of 
mechanical lid elevation for ptosis with crutches or tape but prolonged use leads to exposure keratopathy. Prisms are useful for stable ocular misalignment with mild to moderate but stable ocular misalignment and even lid retraction surgery and strabismus correction through resective surgery can be discussed with the patient. ${ }^{10}$

\section{Acknowledgments}

None.

\section{Conflicts of interest}

The author declares no conflicts of interest.

\section{References}

1. Antonio-Santos AA, Eggenberger ER. Medical treatment options for ocular myasthenia gravis. Curr Opin Ophthalmol. 2008;19(6):468-478.

2. Oosterhuis HJ. Observations of the natural history of myasthenia gravis and the effect of thymectomy. Ann N Y Acad Sci. 1981;377:678-690.

3. Kupersmith MJ, Latkany R, Homel P. Development of generalized disease at 2 years in patients with ocular myasthenia gravis. Arch Neurol. 2003;60(2):243-248.
4. Sommer N, Melms A, Weller M, et al. Ocular myasthenia gravis. A critical review of clinical and pathophysiological aspects. Doc Ophthalmol Adv Ophthalmol. 1993;84(4):309-333.

5. Barton JJ, Fouladvand M. Ocular aspects of myasthenia gravis. Semin Neurol. 2000;20(1):7-20.

6. Nair AG, Patil-Chhablani P, Venkatramani DV, et al. Ocular myasthenia gravis: A review. Indian J Ophthalmol. 2014;62(10):985-991.

7. Padua L, Stalberg E, LoMonaco M, et al. SFEMG in ocular myasthenia gravis diagnosis. Clin Neurophysiol Off J Int Fed Clin Neurophysiol. 2000;111(7):1203-1207.

8. Kelly JJ, Daube JR, Lennon VA, et al. The laboratory diagnosis of mild myasthenia gravis. Ann Neurol. 1982;12(3):238-242.

9. Kerty E, Elsais A, Argov Z, et al. EFNS/ENS Guidelines for the treatment of ocular myasthenia. Eur J Neurol. 2014;21(5):687-693.

10. Bradley EA, Bartley GB, Chapman KL, et al. Surgical correction of blepharoptosis in patients with myasthenia gravis. Ophthal Plast Reconstr Surg. 2001;17(2):103-110. 